University of Wollongong

Research Online

Faculty of Engineering and Information

Faculty of Engineering and Information

Sciences - Papers: Part B

Sciences

2019

Ontological Review of Persuasion Support Systems (PSS) for Health Behavior Change through Physical Activity

Khin Than Win

University of Wollongong, win@uow.edu.au

Arkalgud Ramaprasad

University of Wollongong, University of Illinois at Chicago, aramapra@uow.edu.au

Thant Syn

Texas A \& M International University

Follow this and additional works at: https://ro.uow.edu.au/eispapers1

Part of the Engineering Commons, and the Science and Technology Studies Commons

Research Online is the open access institutional repository for the University of Wollongong. For further information contact the UOW Library: research-pubs@uow.edu.au 


\title{
Ontological Review of Persuasion Support Systems (PSS) for Health Behavior Change through Physical Activity
}

\author{
Abstract \\ Persuasion Support Systems (PSS) for health behavior change can play an important role in promoting \\ health and well-being through physical activity. It is an emerging application at the crossroad between \\ information systems, persuasion, and healthcare. We propose an ontology to systematically and \\ systemically describe the construct of PSS for health behavior change. The ontology deconstructs the \\ construct into its constituent dimensions and elements, and assembles them into a complete, \\ parsimonious description of the same. We then map the corpus of literature on PSS for health behavior \\ change through physical activity onto the ontology. The resulting ontological map highlights the research \\ topics that are highly- and lightly-emphasized, as well as those with little or no emphasis. It illuminates \\ the landscape of research in the corpus; it highlights biases in emphases that can help and hinder the \\ advancement of the corpus. It can be used to develop a roadmap for future research.

\section{Disciplines} \\ Engineering | Science and Technology Studies

\section{Publication Details} \\ Win, K. Than., Ramaprasad, A. \& Syn, T. (2019). Ontological Review of Persuasion Support Systems (PSS) \\ for Health Behavior Change through Physical Activity. Journal of Medical Systems, 43 (3), 49-1-49-12.
}




\section{Ontological Review of Persuasion Support Systems (PSS) for Health Behavior Change through Physical Activity}

Suggested Running Title

PSS for Health Behavior Change 


\begin{abstract}
Persuasion Support Systems (PSS) for health behavior change can play an important role in promoting health and well-being through physical activity. It is an emerging application at the crossroad between information systems, persuasion, and healthcare. We propose an ontology to systematically and systemically describe the construct of PSS for health behavior change. The ontology deconstructs the construct into its constituent dimensions and elements, and assembles them into a complete, parsimonious description of the same. We then map the corpus of literature on PSS for health behavior change through physical activity onto the ontology. The resulting ontological map highlights the research topics that are highly- and lightly-emphasized, as well as those with little or no emphasis. It illuminates the landscape of research in the corpus; it highlights biases in emphases that can help and hinder the advancement of the corpus. It can be used to develop a roadmap for future research.
\end{abstract}

\title{
Introduction
}

Physical activity, fitness, and an active lifestyle are important for the well-being of people young and old, whether they are healthy or having a chronic disease. Moreover, health behavior change, like having regular physical activity, plays an important role in the management of chronic diseases and the prevention of chronic medical conditions. An important objective of healthcare management is to persuade patients and other consumers to undertake targeted behaviors, like physical activity, to improve their health and well-being.

Information technology is billed as a primary driver for improving the welfare and healthcare of aging societies [1,2]. With advances in information technology, information systems can be used to interactively persuade people to modify their physical activity in desirable ways. Many 
previous studies have shown the effectiveness of information systems for behavior modification [3-6]. Computer-mediated lifestyle modification through persuasion and according to the consumers' needs has been identified as one of the strategies of health behavior change [7]. The persuasion can be through tailoring [8-11], communicating better through dialogue support [1214], and providing social support $[15,16]$. Such information systems may be called persuasion support systems (PSS) for health behavior change.

While the literature on PSS for health behavior change through physical activity and other means (for example, medication management) is proliferating, there is not a systemic framework to systematically assess and guide the research. In the absence of such a framework there is a significant risk of bias in the topics researched. Some topics may be heavily emphasized because they are (a) important, (b) in fashion, or (c) convenient for research. On the other hand, some topics may be lightly emphasized because they are (a) unimportant, (b) not in fashion, or (c) inconvenient for research. In addition, there may be topics which have been simply overlooked or are infeasible. These biases can help and hinder the advancement of PSS for health behavior change [17]. They could rapidly advance the domain if the chosen research topics are correct; they could also significantly retard the domain if the topics are incorrect.

In the following, we present an ontological framework for PSS for health behavior change construct. We apply the ontology to map the literature on information systems support for health behavior change through physical activity. We derive the framework by logically deconstructing the construct. We then map the key research articles in the corpus of research onto the ontology to highlight the areas of heavy emphasis, light emphasis, and no emphasis. From the mapping, we (a) paraphrase the themes of research in the domain, and (b) highlight some key themes which have not been addressed in the research. Subsequently, we discuss some possible 
explanations of the biases in inclusion as well as exclusion of topics. Last, we conclude with an agenda for future research on the construct.

\section{Ontology of PSS for Health Behavior Change}

The ontology represents our conceptualization of the domain [18]. It is an "explicit specification of [our] conceptualization,” [19, p. 908] and can be used to systematize the description of the complexity of information systems providing support for persuading health behavior change [20]. The ontology organizes the terminologies and taxonomies of the domain. "Our acceptance of [the] ontology is... similar in principle to our acceptance of a scientific theory, say a system of physics; we adopt, at least insofar as we are reasonable, the simplest conceptual scheme into which the disordered fragments of raw experience can be fitted and arranged.” [21, p. 16]

The challenge is to construct an ontology which is a logical, parsimonious, and a complete description of PSS for health behavior change. It has to be logical in the deconstruction of the domain, and parsimonious yet complete in the representation of the domain. It has to be a closed description of PSS for health behavior change. An existing framework for persuasive systems design [22] was used as a basis for constructing the ontology. The framework is based on an extensive review of the past frameworks and literature. Fogg [23] also identified that computers can play roles as tool, media and social actor for persuasion.

In this paper, we deconstruct PSS for health behavior change into: (a) information systems support for persuasion, (b) the role of persuasion, and (c) the objectives of healthcare. These three are the basic dimensions of the ontology. The underlying argument is that healthy lifestyle and overall improvement in healthcare can be achieved by using information systems support to 
persuade people to adopt healthy behaviors. The argument can be expressed as: health behavioral change is a function of information systems support and persuasion. Thus:

Health behavior change $=\mathrm{f}($ Information Systems Support + Persuasion $)$

Information systems support can aid persuasion in four basic ways. They are through: (a)

structuring the task, (b) dialog support, (c) systemic support, and (d) social support [22]. These four constitute the sub-dimensions of the Information Systems Support dimension. They should be implemented in various features of PSS. Thus:

Information Systems Support $=$ Task Structuring + Dialog Support + System Support + Social Support

Further, based on the literature, each of the four types of support can be expressed in many ways in a system. For example: (a) task structuring can be through reduction, tunneling, tailoring, etc., and (b) social support can be through recognition, facilitation, competition, etc. [22] The many ways of expression of each type of support can be represented by a taxonomy of corresponding functions derived from the literature and practice. A PSS may have one or more functions of each type. Thus:

Task Structuring $\subset[$ Reduction, Tunneling, Tailoring, Personalization, Selfmonitoring, Simulation, Rehearsal]

Dialog Support $\subset[$ Suggestion, Similarity, Praise, Rewards, Liking, Reminders, Support]

System Support $\subset[$ Expertise, Verifiability, Surface Credibility, Real-World Feel, Trustworthiness, Authority, Endorsements] 
Social Support $\subset[$ Recognition, Facilitation, Cooperation, Competition, Learning, Comparison, Influence]

Persuasion is a product of actions intended to reinforce, modify, and change which are focused on knowledge, attitude, and behavior of the person. Transtheoretical model [24] indicates that there are different stages of behavior change, precontemplation, contemplation, preparation, action and maintenance. In the precontemplation and contemplation stage, there is a need to change the behavior and persuasion is to encourage the change. In action stage people need to modify their behavior and in maintenance stage it is to ensure that the person continue the behavior that is adopted and reinforcement is needed. Knowledge, attitude and behavior have been the essential focus in health promotion and these perspectives have been studied widely [25]. Therefore, persuasion can be represented as follows:

Persuasion $=$ Action + Focus

Action $\subset[$ Reinforce, Modify, Change]

Focus $\subset$ [Knowledge, Attitude, Behavior]

Last, the objectives of healthcare are its quality, safety, cost, and parity [26,27].

Health $\subset[$ Quality, Safety, Cost, Parity]

We present the three dimensions with the six sub-dimensions (four of Support and two of Persuasion) pictorially as an ontology of Persuasion Support Systems (PSS) for Health Behavior Change in Figure I. The terms in the ontology are defined in the glossary in Appendix A.

The ontology helps visualize the dimensions, sub-dimensions, elements, and components of PSS for health behavior change systematically and systemically in natural English. Each dimension is 
represented by a single column (Health) or a combination of columns (four for Information System Support, two for Persuasion). The taxonomy of elements constituting each dimension/sub-dimension is listed in the respective column. All the elements together constitute the elements of the system. The columns are connected by adjacent words/phrases — they help concatenate the elements from different columns into phrases/sentences which describe the fragments/components which constitute the system. Thus for example: (a) social recognition to reinforce knowledge to manage cost of care can be a fragment of a system, and (b) task reduction with interactive similarity, system authority, social influence to modify health behavior to manage quality of care can be a component of a system. There are $(7 * 7 * 7 * 7 * 3 * 3 * 4=86,436)$ potential components of the system encapsulated in the ontology. The ontology encapsulates the combinatorial complexity of information systems support for persuading health behavior change.

Figure I

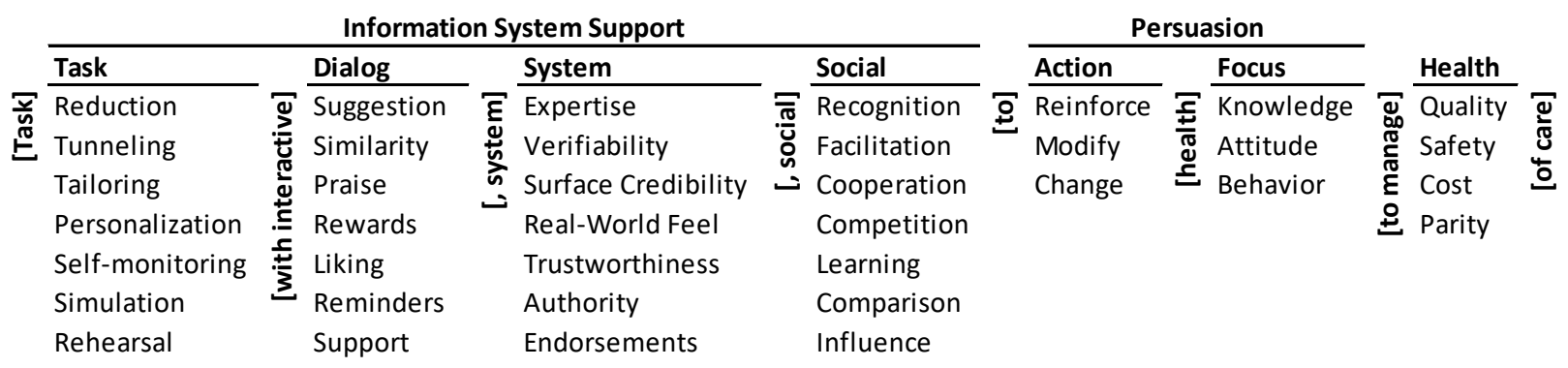

\section{Validity of the Ontology}

The validity of the ontology can be determined "based on questions generated from earlier-stated definitions of an ontology: How explicit is the conceptualization? How logical is the conceptualization? How specific is the conceptualization? How systematic is the description? And how systemic is the description?” [28] In the context of the PSS for health behavior change construct, one may ask: Are the dimensions basic to the domain? Are the taxonomies basic to the 
dimensions? Are the concatenations basic to the domain? We address these questions using the concepts commonly used in social science research: face validity, content validity, systemic validity, and external validity $[29,30]$.

The ontology is logically derived as described earlier. It explicates the underlying logic. In a way, it is a novel reorganization and representation of traditional knowledge from multiple disciplines as synthesized by Oinas-Kukkonen, Harjumaa [22]. Thus, it derives part of its validity from that of the knowledge in the underlying disciplines.

It comprehensively describes PSS for health behavior change using structured natural English understandable to a lay reader. It deconstructs the combinatorial complexity of a complex construct and presents it visually as an easily readable, parsimonious text-table [31]. Listing all the 86,436 components would run into many pages. The ontology encapsulates them in a readable form in less than half a page. Its face validity is high.

Each logical component of PSS for health behavior change derived from the ontology is semantically meaningful as illustrated above; thus, its semantic validity is high. It is easy to ascertain whether a component is instantiated or not. Obversely, it is also easy to ascertain the component that matches an instantiation.

Its dimensions are based on the common frameworks of information systems, persuasion support, and healthcare. The dimension taxonomies include all the basic categories in the common body of knowledge in the respective disciplines. Thus, the content validity of the dimensions, the taxonomies, and the large number of consequent components is high.

Further, the ontology encapsulates all the possible components of a PSS for health behavior change. It has high systemic validity. 


\section{Ontological Review}

We conducted an exhaustive review of published articles on PSS for health behavior change through physical activity. The review team included 4 researchers, namely: a research assistant, two information system academics, and a health informatician. The review was conducted in November 2014. The review team met regularly to ensure that the articles selected were appropriate and complete based on a set of inclusion-exclusion criteria. The following is a detailed description of the process as shown in Figure II which is based on the PRISMA statement for reporting systematic reviews and meta-analyses [32].

\section{Data Sources and Search Strategy}

The articles selected had to examine a computer-based intervention aimed at promoting healthy physical activity as a means of preventing potentially chronic diseases in healthy adults. The platform for these interventions could be a static or mobile computing device, and delivery could be via an application or a browser. The search terms were used to identify potential studies in three databases: PubMed, PsychInfo and Web of Science. These databases cover the domain comprehensively.

To retrieve relevant articles on physical activity, the following search terms were used: (exercise OR motor activity OR sports OR leisure activities) OR (physical* AND active) OR (physical* AND activity) OR (physical* AND activities) OR exercis* OR walking OR cycling OR sport* OR leisure activit* AND (education OR behavior OR behavio* OR education) AND (tailored OR tailoring OR tailor* OR expert system). For the delivery platform, the following search terms were added: (web* OR computer* OR internet* OR online OR mobile OR mHealth OR digital OR technology). We focused on persuasive systems design as a criterion to analyze the data; however, it does not explicitly specified in the search terms. The results are summarized in 
Figure II. PubMed yielded 1,188 articles, PsychInfo 13,577, and Web of Science 30,811. After excluding articles not related to web or mobile technologies, 79, 262, and 640 articles respectively were retained.

Figure II

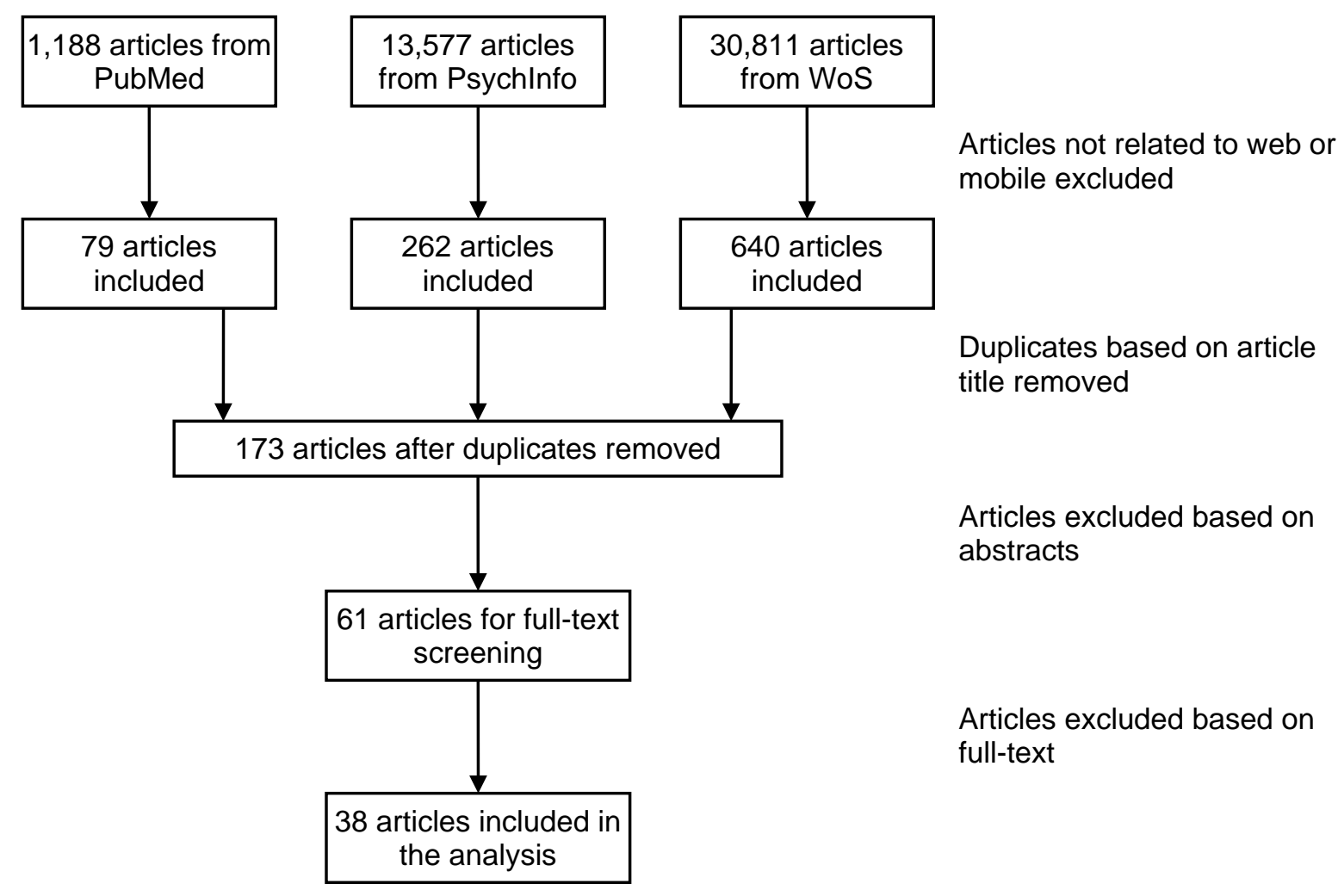

\section{Selection and Exclusion of Studies}

The selection process is shown in Figure II. At the first stage, duplicates and some unrelated articles were eliminated based on titles. A total of 173 articles were included in further screening.

Review, theoretical, and non-English articles were removed from the dataset. Review articles were excluded as they overlapped with original articles on which the reviews were based. Articles discussing only the theoretical framework were excluded due to lack of information of an actual application of PSS. The exclusions were done in two stages. First, exclusions based on 
abstracts resulted in 61 articles. Next, exclusions based on full-texts resulted in a final set of 38 articles.

\section{Data Extraction and Coding}

Detailed information was extracted only for articles that met the aforementioned criteria for inclusion. An Excel spreadsheet was prepared with information of the final 38 articles for coding. Initially, two of the researchers independently coded the articles based on the full-texts. Each coder identified representative sample of text relevant to one or more elements of PSS for health behavior change in each article and mapped it to the ontology. After the round of individual coding, the two coders discussed the discrepancies in their coding and arrived at a consensus for the final coding. All coding adhered strictly to the glossary shown in Appendix A.

The coding by definition is interpretive, based on the semantic interpretation of the text of the article and the glossary by coders knowledgeable about the domain. Its intent was to obtain consensus among the coders through feedback about differences, learning, and (re)interpretation of each article. As such, it is subjective and semantic coding by people, and not objective and syntactic coding by machines or machine-like coders. For these reasons, aggregate measures such as inter-coder reliability (in contrast to article-level similarities and differences) were not considered relevant.

\section{Results}

The ontological map of monads_-individual elements in the ontology—is shown in Figure III. The bar below the category is a visual indicator of the same scaled to the maximum number of occurrences of any one category. The visualization in Figure III clearly highlights the areas of emphases, limited, emphasis, and no emphasis. The map is discussed in detail below. 
Figure III

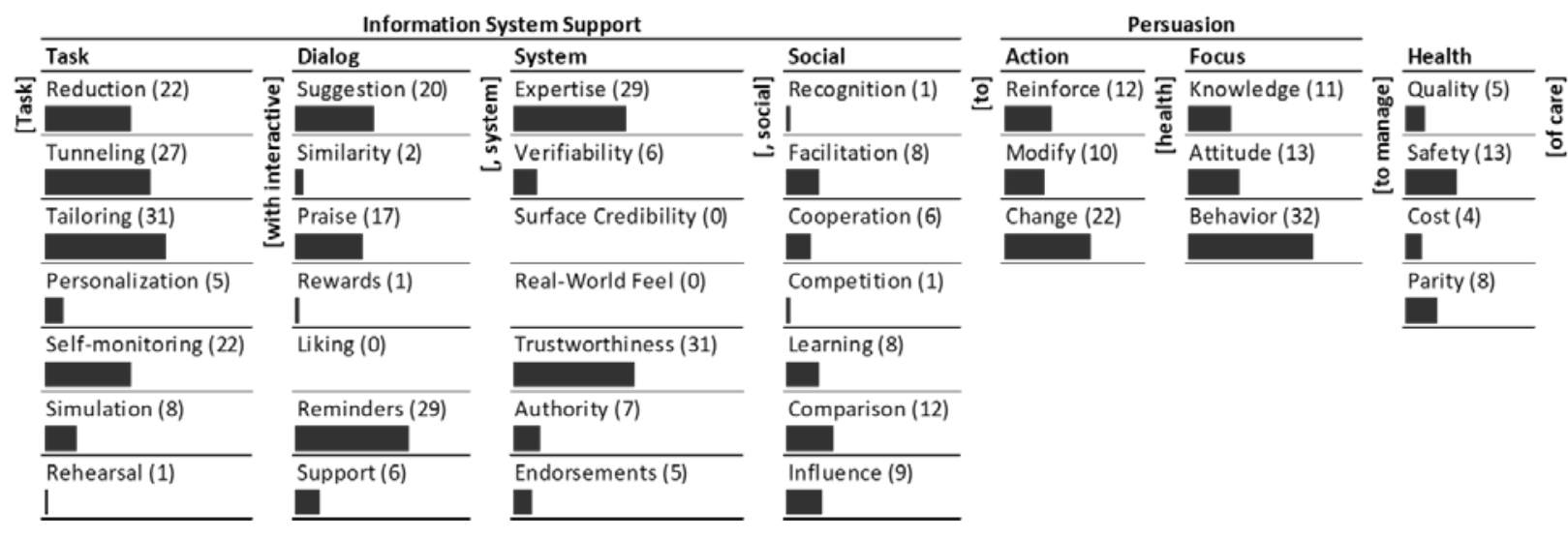

\section{Ontological Map}

The ontological map highlights the 'bright' spots (high frequency), 'light' spots (low frequency), and the 'blind/blank' spots (no frequency) elements in the corpus. In the following, we will analyze the results — the luminosity of each element—with reference to each dimension of the ontology sequentially. (Notes: (1) We will capitalize the words that refer to the dimensions and elements in the ontology, except in describing components. (2) The number in parentheses adjacent to the category indicates its frequency of occurrence in the 38 articles.) Later, the results from the cluster analysis highlight the dominant and not so dominant components—combination of elements across the dimensions.

\section{Task}

Task support features are commonly present in almost all reviewed articles. Tailoring (31) and Tunneling (27) are most frequent, followed by Reduction (22), and Self-monitoring (22). Tailoring was present in the majority of the articles and can be grouped into two types. First, tailoring the program after goal setting - that is allowing the users to choose the options after giving them the available information. Second, after profiling them based on a questionnaire. For example: “after ...a short questionnaire... [participants] immediately received personalized 
physical activity advice on their screen" [33], individual-tailored physical activities "complete a brief survey and immediately receive computer-tailored advice based on their answers” [34], and “the tailored intervention consisted of 'physical activity advice' and an 'action plan”” [35].

Reduction and Tunneling features are incorporated when Tailoring intervention has been provided as tailored information or providing an activity would reduce user effort and bring them to the targeted behavior. Tailoring to the user group is more common than Personalization (5). Simulation (8) and Rehearsal (1) are rarely mentioned.

\section{Dialog}

Reminders (29) is the most frequent feature in the corpus followed by Suggestion (20) and Praise (17). Email and SMS reminders are the most common forms of reminders. Support (6), Similarity (2), and Rewards (1) were mentioned infrequently; Liking (0) was not mentioned at all.

In the reviewed articles, suggestion features are presented as "coached to set useful and realistic goals" [36], "reflect and provide solutions" [36], and "encouraged to seek” [37]. Praise features can be seen in articles giving positive feedback to participants as "on-screen visual and audio positive feedback” [38], “feedback for achievement of their goals” [39], “feedback was designed to provide general encouragement” [40], and "tailored interactive text encouragements" [41].

\section{System}

Among the System attributes for persuasion support, Trustworthiness (31) and Expertise (29) were most frequently discussed in the corpus. Authority (7), Verifiability (6), and Endorsements (5) were infrequently mentioned. Surface Credibility (0) and Real-World Feel (0) were not mentioned at all. 
The articles reviewed describe trustworthiness, expertise, and endorsement features as “supported by research findings and trusted organizations” [34], “appropriate clinically endorsed” [42], "related links" [43], "the behavior-health link" [44], "provided with information on the specific benefits of physical activity (e.g. World Health Organization)” [34], “developed with expert advice, were able to email the exercise specialist questions concerning their program", and "physical activity compared to American College of Sports Medicine (ACSM) recommendations" [35].

\section{Social}

Overall, Social attributes for persuasion support were not discussed as frequently as the Task, Dialog, and System attributes. The most commonly mentioned attribute was Comparison (12). It was followed by Influence (9), Facilitation (8), Learning (8), and Cooperation (6). Recognition (1) and Competition (1) were the least frequent.

Normative influence, social learning, and facilitation are most cited features in social support category in the corpus. Those features were presented in articles as "provide peer support" [45], "share their experiences” [46], "success stories” [43], "a message board to share their experiences” [46] , "enroll a supportive 'buddy”" [47], and "social support among their friends" [48].

\section{Action}

The objective of PSS reviewed here is "health behavior change." Hence, it is not surprising that the dominant Action is Change (22). Interestingly, there is also significant attention paid to Reinforce (12) and Modify (10). Change appears to be considered as part of the continuum of reinforcement-modification-change, rather than as one of the dichotomous states of change/nochange. 
Examples of Reinforcement for continuation of current physical activity include "tips and suggestions will help you” [33], "affirmation through encouragement message," and "keep doing the hard work” [49]. Most studies focused on consumer behavior change towards targeted physical activity.

\section{Focus}

Again, given the objective of PSS on health behavior change, it is not surprising that Behavior (32) is the dominant Focus. However, there is also a significant focus on Attitude (13) and Knowledge (11). There seems to be the recognition of the need to reinforce/modify/change health knowledge/attitude to bring about behavior change—-the ultimate objective of PSS.

\section{Health}

Only a few of the articles explicitly addressed the impact of PSS for health behavior change on Quality, Safety, Cost, and Parity of care. Among those that did the dominant focus is on Safety (13); Parity (8), Quality (5), and Cost (4) are next, in that order.

It can be seen that interventions for health behavior change through physical activities aimed to improve the health outcome for patients and better health management. Safety of the participants have been explicitly mentioned in some articles; for example, these studies excluded seriously ill patients or cancer patients and dialysis patients [50]. Diagnostic questionnaires were included before the intervention for safety in most studies.

Internet-based intervention has reached to socially disadvantaged communities [37] where counselor is not available; for example, the rural and remote area [49] and medically underserved population [51]. Some studies indicated that internet-based intervention would be cost effective only if the study involves a large sample size and a health organization [49]. 


\section{Clusters of Elements}

The clusters of ontology elements in the corpus are shown in Figure IV. The clusters are formed based on the simple matching coefficient (SMC) between pairs of articles in the corpus [52,53]. The SMC is a symmetric measure which is based on the similarity of the ontological elements included (coded as ' 1 ') and not included (coded as ' 0 ') in the articles. The grouping was based on the nearest neighbor between the clusters. The clusters in Figure IV clearly highlight groups of elements emphasized heavily in the corpus, and groups emphasized lightly or not at all. The clusters are discussed in greater detail below.

Figure IV

\begin{tabular}{|c|c|c|c|c|c|c|c|c|c|c|}
\hline \multicolumn{6}{|c|}{ Information System Support } & \multicolumn{3}{|c|}{ Persuasion } & \multirow{2}{*}{\multicolumn{2}{|c|}{ Health }} \\
\hline & Task & Dialog & System & & Social & Action & & Focus & & \\
\hline$\frac{\bar{y}}{\mathrm{~g}}$ & Reduction & $\underset{\mathcal{D}}{\mathrm{g}}$ Suggestion & $\bar{E}$ Expertise & $\frac{7}{0}$ & Recognition & Reinforce & 祬 & Knowledge & $\widetilde{\Xi}^{\circ}$ Quality & 疍 \\
\hline & Tunneling & Similarity & Wै Verifiability & 。̆ & Facilitation & Modify & ฮू & Attitude & Safety & 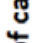 \\
\hline & Tailoring & $\frac{\pi}{0}$ Praise & के Surface Credibility & $\vec{\Xi}$ & Cooperation & Change & & Behavior & हึ Cost & \\
\hline & Personalization &.$\cong$ Rewards & Real-World Feel & & Competition & & & & \& Parity & \\
\hline & Self-monitoring & E Liking & Trustworthiness & & Learning & & & & & \\
\hline & Simulation & 3 Reminders & Authority & & Comparison & & & & & \\
\hline & Rehearsal & Support & Endorsements & & Influence & & & & & \\
\hline & Cluster 1 - Primar & & & & & & & & & \\
\hline & Cluster 2 - Sec & & & & & & & & & \\
\hline & Clus & & & & & & & & & \\
\hline
\end{tabular}

Three clusters of information system support for health behavior change through physical activity can be seen in Figure IV. The primary cluster which encapsulates the dominant theme of the corpus can be expressed as:

Task reduction/tunneling/tailoring/self-monitoring with interactive suggestion/reminders, system expertise/trustworthiness to change health behavior. 
The less dominant theme of the corpus is captured in the secondary cluster. It can be stated as:

Task simulation with interactive praise to reinforce/modify health knowledge/attitude to manage safety/parity of care.

The last, tertiary cluster depicts the non-dominant or absent theme of the corpus. The theme can be described as:

Task personalization/rehearsal with interactive similarity/rewards/liking/support, system verifiability/surface credibility/real-word feel/authority/endorsements, social recognition/facilitation/cooperation/competition/learning/comparison/ influence to manage quality/cost of care.

\section{Discussion}

Most of the articles in the corpus focus explicitly on Information System Support and Persuasion, but not their Health outcome. Further, no Health element is part of the primary cluster in Figure IV. This is surprising because the ultimate objective of PSS for health behavior change through physical activity is to improve the health of the subjects. Even if the health behavior changes through physical activity, the eventual impact on the health of the individuals, hence the efficacy of PSS, may remain unclear. As article search is not limited to the clinical trial and the outcome studies only, there is a possibility that the proportion of the published articles are more on the design perspectives and usability of technology and not on the effectiveness studies on health. In other words, that highlights that a lot of applications were developed and discussed more on the design perspectives and not many health outcome studies conducted were more on whether there is increased in physical activity only but not the actual health outcome. 
Within Information System Support, the dominant emphasis is on the Task, Dialog, and System support. There is relatively less emphasis on Social support. Given the lay discussion on the importance of social support, the lack of emphasis on it is surprising. It is envisioned that it could be more system providing social support categories in recent year with the emergence of widespread use of social media. Reviewed articles also indicated that the system is developed as a hybrid system. That is while the task support of tailored messages and reminders features are present in the system, face to face communication also was included as part of those study and social support in provided through direct communication with the healthcare provider in person rather than through the technology.

Five out of seven Task elements belong to the primary or secondary cluster of Information System Support elements, but only three of the seven Dialog elements and two of the seven System elements. No element of Social support is part of either cluster. There may be many reasons for inclusion/exclusion of these elements from Information System Support. Most of the articles focused on the description of the intervention using PSS instead of describing the look and feel of PSS in detail. Consequently, two System support features, Surface Credibility and Real-World Feel, were not explicitly addressed although the actual systems studied may include these features. Similarly, support for personalization of tasks was scarcely mentioned in the articles. Personalized feedback information described in some articles were mostly designed for tailoring — “providing tailored feedback to user groups" rather than "personalized content and services for user" [54]. Rehearsal is another Task support element not well-represented in the reviewed articles. One possible use of rehearsal features is in the interactive applications for physical activity such as virtual games (for example, WiiU) where users can practice the behavior. This indicates that some PSS features may be application-dependent. Similarly, most 
articles in the corpus are health intervention studies based on randomized controlled-trials. PSS identified in these articles focused on Task (Tailoring, Tunneling, Self-monitoring), Dialog (Praise, Reminder, Suggestion), and System (Trustworthiness, Expertise) support. Social support, on the other hand, was not emphasized as much as other PSS support features. The landscape might change in the future studies as more comprehensive applications have been developed recently. Some of the studies from the review included the social support as the system was part of the system of face to face communication. Including studies in virtual reality related physical activity studies would also provide the PSS for virtual reality application but these applications were not included in the current study.

Within Persuasion, there is slightly greater focus on Focus than on Action. All the elements of the two dimensions of Persuasion belong to the primary or secondary cluster. There is only a difference in emphases but not the exclusion of elements. Persuasion support can be provided to users through different media and intervention. Current review indicated that although the behavior change could be seen in the primary cluster, knowledge and attitude could be seen in the secondary cluster. Proving information to users play an important role in persuasion as if users are unaware of the importance of change they would not likely to change their behavior. Knowledge attitude behavior model and the information motivation behavior model [55] indicated the importance of the information and knowledge. Health belief model [56] also indicated that user's perceived belief and attitude play an important role for health behavior change. PSD has identified the design features for information system support and that needs to be considered with persuasion action and support as presented in the PSS. Having the information and knowledge or education regarding the importance of physical activity will assist 
consumers towards their target behavior change [57]. Thus, specific knowledge related to the needs of physical activity should be presented in the PSS.

Although the Health dimension is less emphasized, two of its elements—Safety and Parity—are part of the secondary cluster. Quality and Cost are excluded from both clusters. It must be noted that the coding was based on the information presented explicitly in the articles. Hence, some dimensions that may generally be inferred, but not specifically stated, were not coded. For instance, one may argue that interventions for health behavior change are inherently intended to improve quality and safety of healthcare of users, and PSS is expected to improve personal health management by promoting self-management behavior, which may also likely promote healthy living and reduce healthcare cost. Nonetheless, if an article did not explicitly indicate that quality, cost, safety, or parity was one of the goals of the study, none of these elements was coded to reduce the effect of biases based on a coder's prior experience.

Thus, the ontological mapping of the corpus reveals a few frequently-trod pathways to the use of PSS for health behavior change through physical activity, but not necessarily to improved health. The mapping also reveals many potential untrod pathways to the realization of the same outcome. Some of the latter pathways may be infeasible; but many may be innovative and help improve the effectiveness of PSS. The ontological review is based on clinical trial studies and most studies examined at a limited time period. Long term motivation for life style changes would need to include those features in primary cluster and secondary cluster as well as features from social support such as social learning, social comparison, social influence etc. It could be seen that ontological review in the behavior change of physical activity studies target to include a few features in PSS. Having an ontology of PSS for behavior change could be the basis for future 
development, assisting system designers and professionals to design a health behavior change support system.

\section{Conclusion}

Persuasive system support (PSS) for health behavior change through physical activity is an important, growing research domain. It needs a systemic framework to map and assess the extant research systematically, and to guide future research. Oinas-Kukkonen, Harjumaa [54] provide the starting point for such a framework in their synthesis of key issues, process models, and system features in persuasive systems design. We draw upon their framework, combine it with

underlying dimensions of persuasion, and the commonly sought healthcare outcomes to develop and ontology of PSS for heath behavior change. In this paper, we apply the framework to study such change through physical activity.

We obtained 38 articles in the domain through an exhaustive search and selection process. The ontological map of these articles highlights the areas of heavy, light, and no emphasis in the domain.

The potential of the domain, as expressed in the ontology, has not been exploited systematically by the research in the domain. There is relatively little explicit attention in the research to the healthcare outcomes in terms of quality, safety, cost, and parity. Behavior change by itself may have little value in the overall context of healthcare unless it can be shown to affect the primary outcomes sought through such care. The paucity of research neither affirms nor denies the ultimate effect on healthcare-it highlights the need to study these effects explicitly.

Persuasion focused on reinforcement and modification of knowledge and attitude is given relatively less attention compared to research on change of behavior. Reinforcement and 
modification complement change. By the same token, persuasion of people about their knowledge and attitude complements, usually precedes, persuasion regarding behavior. Managing these complementarities will play an important role in the effectiveness of PSS.

Last, there are many task-, interactive dialog-, system-, and social support-based methods that have not been explored intensively. Further, among the four types of task-support has been explored most intensively and social-support least intensively. All types of support need to be explored systematically to improve the effectiveness of PSS.

Thus, the ontological review of PSS for health behavior change through physical activity paints the portrait of a domain whose research is rich but can be enriched in many ways. First, it can be enriched by linking it to specific healthcare outcomes. Second, it can be enriched by exploring the less-explored and unexplored methods of support and persuasion. Third, and last, by approaching the problem systemically using the ontology.

\section{Compliance with Ethical Standards:}

Funding: no funding

Conflict of Interest: All authors declare that there is no conflict of interest.

Ethical approval: This article does not contain any studies with human participants or animals performed by any of the authors.

\section{References}

1. Klecun-Dabrowska E, Cornford T (2000) Telehealth acquires meanings: information and communication technologies within health policy. Inform Syst J 10 (1):41-63. doi:10.1046/j.1365-2575.2000.00074.x 
2. Boonstra A, Van Offenbeek M (2010) Towards consistent modes of e-health implementation: structurational analysis of a telecare programme's limited success. Inform Syst J 20 (6):537-561. doi:10.1111/j.1365-2575.2010.00358.x

3. Chatterjee S, Price A (2009) Healthy Living with Persuasive Technologies: Framework, Issues, and Challenges. J Am Med Informat Assoc 16 (2):171-178. doi:10.1197/jamia.M2859 4. Kaptein M, Markopoulos P, de Ruyter B, Aarts E (2015) Personalizing persuasive technologies: Explicit and implicit personalization using persuasion profiles. Int J HumanComputer Stud 77:38-51. doi:10.1016/j.ijhcs.2015.01.004

5. Daud NA, Sahari@Ashaari N, Muda Z (2013) An Initial Model of Persuasive Design in Web based Learning Environment. Procedia Tech 11:895-902. doi:10.1016/j.protcy.2013.12.273 6. Looije R, Neerincx MA, Cnossen F (2010) Persuasive robotic assistant for health selfmanagement of older adults: Design and evaluation of social behaviors. Int J Human-Computer Stud 68 (6):386-397. doi:10.1016/j.ijhcs.2009.08.007

7. Matthews J, Win KT, Oinas-Kukkonen H, Freeman M (2016) Persuasive Technology in Mobile Applications Promoting Physical Activity: a Systematic Review. J Med Syst 40 (3):72. doi:10.1007/s10916-015-0425-x

8. Morrison LG, Yardley L, Powell J, Michie S (2012) What Design Features Are Used in Effective e-Health Interventions? A Review Using Techniques from Critical Interpretive Synthesis. Telemed e-Health 18 (2):137-144. doi:10.1089/tmj.2011.0062

9. Doupi P, van der Lei J (2005) Design and implementation considerations for a personalized patient education system in burn care. Int J Med Informat 74 (2-4):151-157. doi:10.1016/j.ijmedinf.2004.04.021 
10. Clayman ML, Boberg EW, Makoul G (2008) The use of patient and provider perspectives to develop a patient-oriented website for women diagnosed with breast cancer. Patient Educ Counseling 72 (3):429-435. doi:10.1016/j.pec.2008.05.032

11. Win KT, Hassan NM, Oinas-Kukkonen H, Probst Y (2016) Online Patient Education for Chronic Disease Management: Consumer Perspectives. J Med Syst 40 (4):88. doi:10.1007/s10916-016-0438-0

12. Ferney SL, Marshall AL (2006) Website physical activity interventions: preferences of potential users. Health Educ Res 21 (4):560-566. doi:10.1093/her/cyl013

13. DeGuzman MA, Ross MW (1999) Assessing the application of HIV and AIDS related education and counselling on the Internet. Patient Educ Counseling 36 (3):209-228. doi:10.1016/S0738-3991(98)00096-2

14. Gosselin P, Poitras P (2008) Use of an internet "viral" marketing software platform in health promotion. J Med Internet Res 10 (4):e47. doi:10.2196/jmir.1127

15. Rezailashkajani M, Roshandel D, Ansari S, Zali MR (2008) A web-based patient education system and self-help group in Persian language for inflammatory bowel disease patients. Int $\mathrm{J}$ Med Informat 77 (2):122-128. doi:10.1016/j.ijmedinf.2006.12.001

16. Yamout SZ, Glick ZA, Lind DS, Monson RAZ, Glick PL (2011) Using social media to enhance surgeon and patient education and communication. Bull Am College Surgeons 96 (7):715

17. Ramaprasad A, Syn T (2014) Strong and Meaningful Use of Healthcare Information Systems (HIS). In: Bienkiewicz M, Verdier C, Plantier G, Schultz T, Fred A, Gamboa H (eds) Proceedings of the International Conference on Health Informatics (BIOSTEC 2014). SciTePress, Angers, Loire Valley, France, pp 381-386. doi:10.5220/0004870303810386 
18. Gruber TR (2008) Ontology. In: Liu L, Özsu MT (eds) Encyclopedia of Database Systems. Springer-Verlag, New York, pp 1963-1965

19. Gruber TR (1995) Toward Principles for the Design of Ontologies Used for Knowledge Sharing. Int J Human-Computer Stud 43 (5-6):907-928. doi:10.1006/ijhc.1995.1081

20. Cimino JJ (2006) In defense of the Desiderata. J Biomedical Informat 39 (3):299-306. doi:10.1016/j.jbi.2005.11.008

21. Quine WVO (1961) From a Logical Point of View. Second, revised edn. Harvard University Press, Boston, MA, USA

22. Oinas-Kukkonen H, Harjumaa M (2008) Towards deeper understanding of persuasion in software and information systems. In: Proceedings of the 1st International Conference on Advances in Computer-Human Interaction. IEEE, pp 200-205

23. Fogg BJ (2003) Persuasive Technology: Using Computers to Change What We Think and Do. Persuasive Technology. Morgan Kaufmann, San Francisco. doi:10.1016/B978-1-55860-6432.X5000-8

24. Prochaska JO, Butterworth S, Redding CA, Burden V, Perrin N, Leo M, Flaherty-Robb M, Prochaska JM (2008) Initial efficacy of MI, TTM tailoring and HRI's with multiple behaviors for employee health promotion. Preventive Medicine 46 (3):226-231. doi:https://doi.org/10.1016/j.ypmed.2007.11.007

25. Malik NA, Zhang J, Lam OLT, Jin L, McGrath C (2017) Effectiveness of computer-aided learning in oral health among patients and caregivers: a systematic review. J Am Med Informat Assoc 24 (1):209-217. doi:10.1093/jamia/ocw045

26. Ramaprasad A, Syn T, Thirumalai M (2014) An Ontological Map for Meaningful Use of Healthcare Information Systems (MUHIS). In: Bienkiewicz M, Verdier C, Plantier G, Schultz T, 
Fred A, Gamboa H (eds) Proceedings of the International Conference on Health Informatics (BIOSTEC 2014). SciTePress, Angers, Loire Valley, France, pp 16-26.

doi:10.5220/0004734500160026

27. Ramaprasad A, Syn T, Thirumalai M (2015) Ontological Analysis of Meaningful Use of Healthcare Information Systems (MUHIS) Requirements and Practice. In: Plantier G, Schulz T, Fred A, Gamboa H (eds) Biomedical Engineering Systems and Technologies, vol 511.

Communications in Computer and Information Science. Springer International Publishing, Switzerland, pp 315-330. doi:10.1007/978-3-319-26129-4_21

28. Ramaprasad A, Syn T (2015) Ontological Meta-Analysis and Synthesis. Comm Assoc Inform Syst 37 (7):138-153

29. Brennan L, Voros J, Brady E (2011) Paradigms at play and implications for validity in social marketing research. J Soc Market 1 (2):100-119. doi:10.1108/20426761111141869

30. Horn BR, Lee I-H (1989) Toward integrated interdisciplinary information and communication sciences: a general systems perspective. In: Proceedings of the 22nd Hawaii International Conference on System Sciences (HICSS 1989), vol 4. IEEE, Kailua-Kona, Hawaii, USA, pp 244-255. doi:10.1109/HICSS.1989.48129

31. Tufte ER (1990) Envisioining Information. Graphics Press, Cheshire, CT

32. Liberati A, Altman DG, Tetzlaff J, Mulrow C, Gøtzsche PC, Ioannidis JP, Clarke M, Devereaux P, Kleijnen J, Moher D (2009) The PRISMA Statement for Reporting Systematic Reviews and Meta-Analyses of Studies That Evaluate Health Care Interventions: Explanation and Elaboration. PLoS Med 6 (7). doi:10.1371/journal.pmed.1000100 
33. Ammann R, Vandelanotte C, de Vries H, Mummery WK (2013) Can a Website-Delivered Computer-Tailored Physical Activity Intervention Be Acceptable, Usable, and Effective for Older People? Health Educ Behav 40 (2):160-170. doi:10.1177/1090198112461791 34. Alley S, Jennings C, Plotnikoff RC, Vandelanotte C (2014) My Activity Coach - Using video-coaching to assist a web-based computer-tailored physical activity intervention: a randomised controlled trial protocol. BMC Publ Health 14. doi:10.1186/1471-2458-14-738 35. Spittaels H, De Bourdeaudhuij I, Brug J, Vandelanotte C (2007) Effectiveness of an online computer-tailored physical activity intervention in a real-life setting. Health Educ Res 22 (3):385-396. doi:10.1093/her/cyl096

36. Kelders SM, van Gemert-Pijnen JE, Werkman A, Seydel ER (2010) Evaluation of a webbased lifestyle coach designed to maintain a healthy bodyweight. J Telemed Telecare 16 (1):3-7. doi:10.1258/jtt.2009.001003

37. King AC, Bickmore TW, Campero MI, Pruitt LA, Yin JL (2013) Employing Virtual Advisors in Preventive Care for Underserved Communities: Results From the COMPASS Study. J Health Comm 18 (12):1449-1464. doi:10.1080/10810730.2013.798374

38. Winett RA, Anderson ES, Wojcik JR, Winett SG, Bowden T (2007) Guide to Health: Nutrition and Physical Activity Outcomes of a Group-Randomized Trial of an Internet-Based Intervention in Churches. Ann Behav Med 33 (3):251-261. doi:10.1007/BF02879907 39. Antypas K, Wangberg SC (2014) An Internet- and Mobile-Based Tailored Intervention to Enhance Maintenance of Physical Activity After Cardiac Rehabilitation: Short-Term Results of a Randomized Controlled Trial. J Med Internet Res 16 (3):78-95. doi:10.2196/jmir.3132 40. Morgan PJ, Callister R, Collins CE, Plotnikoff RC, Young MD, Berry N, McElduff P, Burrows T, Aguiar E, Saunders KL (2013) The SHED-IT Community Trial: A Randomized 
Controlled Trial of Internet- and Paper-Based Weight Loss Programs Tailored for Overweight and Obese Men. Ann Behav Med 45 (2):139-152. doi:10.1007/s12160-012-9424-z

41. Klausen SH, Mikkelsen UR, Hirth A, Wetterslev J, Kjaergaard H, Sondergaard L, Andersen LL (2012) Design and rationale for the PREVAIL study: Effect of e-Health individually tailored encouragements to physical exercise on aerobic fitness among adolescents with congenital heart disease—a randomized clinical trial. Am Heart J 163 (4):549-556. doi:10.1016/j.ahj.2012.01.021 42. Hearn L, Miller M, Fletcher A (2013) Online healthy lifestyle support in the perinatal period: what do women want and do they use it? Aust J Primary Health 19 (4):313-318. doi:10.1071/py13039

43. Brindal E, Freyne J, Saunders I, Berkovsky S, Smith G, Noakes M (2012) Features Predicting Weight Loss in Overweight or Obese Participants in a Web-Based Intervention: Randomized Trial. J Med Internet Res 14 (6):114-129. doi:10.2196/jmir.2156 44. Ezendam N, Noordegraaf V, Kroeze W, Brug J, Oenema A (2013) Process evaluation of FATaintPHAT, a computer-tailored intervention to prevent excessive weight gain among Dutch adolescents. Health Promot Int 28 (1):26-35. doi:10.1093/heapro/das021

45. Mitchell BL, Lewis NR, Smith AE, Rowlands AV, Parfitt G, Dollman J (2014) Rural Environments and Community Health (REACH): a randomised controlled trial protocol for an online walking intervention in rural adults. BMC Publ Health 14. doi:10.1186/1471-2458-14-969 46. Hurling R, Catt M, De Boni M, Fairley BW, Hurst T, Murray P, Richardson A, Sodhi JS (2007) Using Internet and Mobile Phone Technology to Deliver an Automated Physical Activity Program: Randomized Controlled Trial. J Med Internet Res 9 (2):1-12. doi:10.2196/jmir.9.2.e7 
47. Rothert K, Strecher VJ, Doyle LA, Caplan WM, Joyce JS, Jimison HB, Karm LM, Mims AD, Roth MA (2006) Web-based Weight Management Programs in an Integrated Health Care Setting: A Randomized, Controlled Trial. Obes 14 (2):266-272. doi:10.1038/oby.2006.34 48. Patrick K, Marshall SJ, Davila EP, Kolodziejczyk JK, Fowler JH, Calfas KJ, Huang JS, Rock CL, Griswold WG, Gupta A, Merchant G, Norman GJ, Raab F, Donohue MC, Fogg BJ, Robinson TN (2014) Design and implementation of a randomized controlled social and mobile weight loss trial for young adults (project SMART). Contemp Clin Trials 37 (1):10-18. doi:10.1016/j.cct.2013.11.001

49. Funk KL, Stevens VJ, Bauck A, Brantley PJ, Hornbrook M, Jerome GJ, Myers VH, Appel L (2011) Development and Implementation of a Tailored Self-assessment Tool in an Internet-based Weight Loss Maintenance Program. Clin Pract Epidemiol Ment Health 7:67-73. doi:10.2174/1745017901107010067

50. Parekh S, King D, Boyle FM, Vandelanotte C (2014) Randomized controlled trial of a computer-tailored multiple health behaviour intervention in general practice: 12-month followup results. Int J Behav Nutr Phys Activity 11. doi:10.1186/1479-5868-11-41

51. Martinez JL, Duncan LR, Rivers SE, Latimer AE, Salovey P (2013) Examining the use of message tailoring to promote physical activity among medically underserved adults. J Health Psychol 18 (4):470-476. doi:10.1177/1359105312445798

52. Cheetham AH, Hazel JE (1969) Binary (Presence-Absence) Similarity Coefficients. J Paleontol 43 (5):1130-1136. doi:10.2307/1302424

53. Gower JC (1971) A General Coefficient of Similarity and Some of Its Properties. Biometrics 27 (4):857-871. doi:10.2307/2528823 
54. Oinas-Kukkonen H, Harjumaa M (2009) Persuasive Systems Design: Key Issues, Process Model, and System Features. Comm Assoc Inform Syst 24:485-500

55. Fisher CM (2012) Adapting the Information-Motivation-Behavioral Skills Model:Predicting HIV-Related Sexual Risk Among Sexual Minority Youth. Health Education \& Behavior 39 (3):290-302. doi:10.1177/1090198111406537

56. Ajzen I, Fishbein M (1980) Understanding attitudes and predicting social behaviour. Prentice-Hall, Englewood Cliffs, N.J.

57. Win KT, Hassan N, Bonney A, Iverson D (2015) Benefits of Online Health Education: Perception from Consumers and Health Professionals. J Med Syst 39 (3):1-8. doi:10.1007/s10916-015-0224-4 


\section{Appendix A: Glossary}

Information System Support: Support provided by persuasive support systems Task:

Reduction: Reduce effort users expend when performing target behavior

Tunneling: Guide users in attitude change by providing means for action that brings them closer to target behavior

Tailoring: Provide tailored info for user groups

Personalization: Offer personalized content and services for users

Self-monitoring: Provide means for users to track their performance or status

Simulation: Provide means for observing link between cause \& effect with regard to users' behavior

Rehearsal: Provide means for rehearsing target behavior

Dialog:

Suggestion: Suggest users carry out behaviors while using the system

Similarity: Imitate its users in some specific way

Praise: Use praise via words, images, symbols, sounds to provide user feedback based on behaviors

Rewards: Provide virtual rewards for users to give credit for performing target behavior

Liking: Have a look \& feel that appeals to users

Reminders: Remind users of their target behavior while using the system

Support: Adopt a social role

System:

Expertise: Provide info showing knowledge, experience \& competence

Verifiability: Provide means to verify accuracy of site content via outside sources

Surface Credibility: Have competent look \& feel

Real-World Feel: Provide info of the organization/actual people behind it content \& services

Trustworthiness: Provide info that is truthful, fair \& unbiased

Authority: Refer to people in the role of authority

Endorsements: Provide endorsements from respected sources

Social:

Recognition: Provide public recognition for users who perform their target behavior

Facilitation: Provide means for discerning others who are performing the behavior

Cooperation: Provide means for co-operation

Competition: Provide means for competing with others

Learning: Provide means to observe others performing their target behaviors to see outcome of their behavior

Comparison: Provide means for comparing performance with the performance of others

Influence: Provide means for gathering people who have same goal \& make them feel norms

Persuasion: Persuade the user

Action: Persuasive action of the system

Reinforce: Reinforce the object of persuasion

Modify: Modify the object of persuasion

Change: Change the object of persuasion 
Focus: Focus of the persuasion

Knowledge: Knowledge of the user

Attitude: Attitude of the user

Behavior: Behavior of the user

Health: Outcomes of healthcare of users

Quality: Quality of healthcare of users

Safety: Safety of healthcare of users

Cost: Cost of healthcare of users

Parity: Parity of healthcare of users 